\title{
Spectroscopic link between adsorption site occupation and local surface chemical reactivity
}

Baraldi, A.; Lizzit, S.; Comelli, G.; Kiskinova, M.; Rosei, R.; Honkala, Johanna Karoliina; Nørskov, Jens Kehlet

Published in:

Physical Review Letters

Link to article, DOI:

10.1103/PhysRevLett.93.046101

Publication date:

2004

Document Version

Publisher's PDF, also known as Version of record

Link back to DTU Orbit

Citation (APA):

Baraldi, A., Lizzit, S., Comelli, G., Kiskinova, M., Rosei, R., Honkala, J. K., \& Nørskov, J. K. (2004).

Spectroscopic link between adsorption site occupation and local surface chemical reactivity. Physical Review Letters, 93(4), 046101. https://doi.org/10.1103/PhysRevLett.93.046101

\section{General rights}

Copyright and moral rights for the publications made accessible in the public portal are retained by the authors and/or other copyright owners and it is a condition of accessing publications that users recognise and abide by the legal requirements associated with these rights.

- Users may download and print one copy of any publication from the public portal for the purpose of private study or research.

- You may not further distribute the material or use it for any profit-making activity or commercial gain

- You may freely distribute the URL identifying the publication in the public portal 


\title{
Spectroscopic Link between Adsorption Site Occupation and Local Surface Chemical Reactivity
}

\author{
A. Baraldi, ${ }^{1,2}$ S. Lizzit,${ }^{3}$ G. Comelli,,${ }^{1,2}$ M. Kiskinova, ${ }^{3}$ R. Rosei, ${ }^{1,2, *}$ K. Honkala, ${ }^{4}$ and J. K. Nørskov ${ }^{3}$ \\ ${ }^{1}$ Dipartimento di Fisica, Universitá di Trieste, Via Valerio 2, 34127 Trieste, Italy \\ ${ }^{2}$ Laboratorio T.A.S.C.-I.N.F.M., Strada Statale 14 Km 163.5, 34012 Trieste, Italy \\ ${ }^{3}$ Sincrotrone Trieste, Strada Statale $14 \mathrm{Km}$ 163.5, 34012 Trieste, Italy \\ ${ }^{4}$ Center for Atomic-scale Materials Physics (CAMP), Department of Physics, Technical University of Denmark, \\ DK-2800 Lyngby, Denmark
}

(Received 21 October 2003; published 20 July 2004)

\begin{abstract}
In this Letter we show that sequences of adsorbate-induced shifts of surface core level (SCL) X-ray photoelectron spectra contain profound information on surface changes of electronic structure and reactivity. Energy shifts and intensity changes of time-lapsed spectral components follow simple rules, from which adsorption sites are directly determined. Theoretical calculations rationalize the results for transition metal surfaces in terms of the energy shift of the $d$-band center of mass and this proves that adsorbate-induced SCL shifts provide a spectroscopic measure of local surface reactivity.
\end{abstract}

DOI: 10.1103/PhysRevLett.93.046101

PACS numbers: 68.43.Fg, 79.60.-i, 82.45.Jn

It has long been known that many transition metals behave as selective and highly efficient catalysts for a large number of industrially relevant chemical reactions. One of the most important long-term goals of surface science is to provide a thorough understanding, at the atomic level, of the complex phenomena taking place at the surface of such catalysts [1-3]. The chemical reactivity of catalytically active sites on surfaces is determined by the local electronic structure, which in turn, depends on local geometry and atomic composition [4]. To design surfaces with prescribed catalytic properties, it is crucial to develop experimental methods allowing the determination of adsorption sites and their time evolution in the presence of reactants, while simultaneously giving a measure of the local surface reactivity. An experimental technique with these capabilities would have a far reaching impact in such diverse fields as surface physics and technology and chemical and biological catalysis and could lead to a decisive step towards the possibility of designing novel catalytic materials.

Here we show that sequences of high resolution x-ray photoelectron spectroscopy surface core level (SCL) spectra [5], obtained in situ during a chemisorption experiment on transition metals, contain a detailed description of the geometrical structure of adsorption sites and represent fingerprints of the instantaneous configuration of populations of adsorbates which evolve on the surface. Density functional theory (DFT) calculations allow us to rationalize and generalize these results in terms of the surface atom-projected $d$-band center of the metal substrate and to correlate SCLs to local changes of surface chemical reactivity [4].

In Fig. 1(a) we report the surface components of a selection of rhodium $3 d_{5 / 2}$ core level spectra measured in situ [6] at the SuperESCA beam line (ELETTRA) at three different oxygen coverages, during oxygen adsorp- tion on $\mathrm{Rh}(100)$. The spectra (chosen from a long sequence) have been fitted to show the different surface components that arise during oxygen exposure [7]. The bulk peak, which remains essentially unchanged (and is not relevant to our discussion), has been omitted for simplicity. The starting condition of the experiment is an atomically clean $\mathrm{Rh}(100)$ surface with a single surface peak $\left(\mathrm{Rh}_{0}\right)$ at a binding energy shift of $-645 \pm 20 \mathrm{meV}$, with respect to the bulk component. Oxygen adsorption leads to the appearance of a new core level component at a

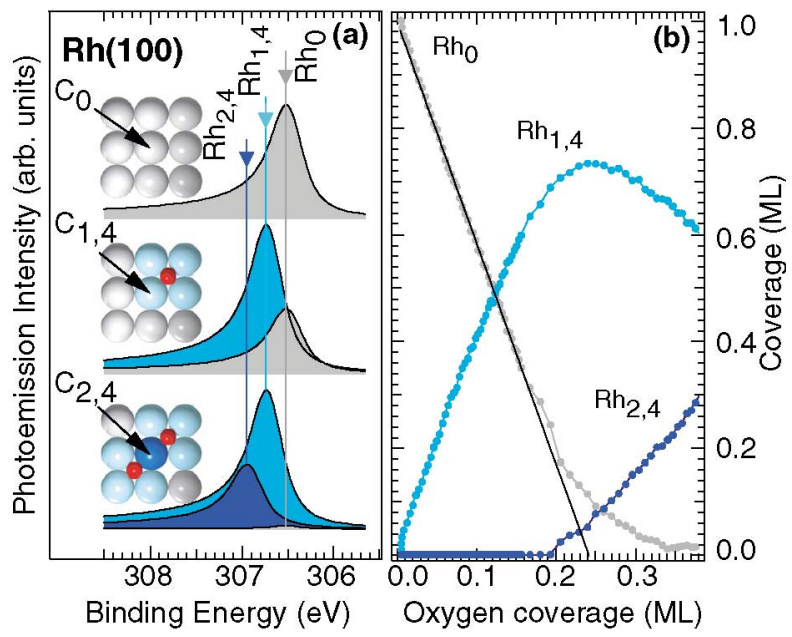

FIG. 1 (color). (a) Surface components of the Rh3 $d_{5 / 2}$ core level spectra for $\mathrm{O} / \mathrm{Rh}(100)$ at $\Theta_{0}=0,0.185$, and $0.375 \mathrm{ML}$ oxygen coverages. The gray component $\mathrm{Rh}_{0}$ originates from the clean surface $\mathrm{Rh}$ atoms, while $\mathrm{Rh}_{1,4}$ and $\mathrm{Rh}_{2,4}$ peaks originate from surface $\mathrm{Rh}$ atoms which bind to one and two oxygen atoms, respectively. (b) Oxygen coverage dependence of the three peaks' integrated areas, obtained by fitting a sequence of spectra (recorded in situ during the uptake experiment). The areas have been normalized to the initial clean peak area. 
shift of $-425 \pm 20 \mathrm{meV}$. Since at low coverage oxygen adsorbs on this surface on fourfold sites [8], we denote this adsorption configuration as $\mathrm{C}_{1,4}$ and the new peak as $\mathrm{Rh}_{1,4}$. In this notation the first index represents the number of adparticles to which the substrate atom is bound, while the second denotes the number of substrate atoms to which the adparticle is bound.

Upon increasing the oxygen coverage, a third surface component appears while the $p(2 \times 2)-\mathrm{O}$ ordered structure starts converting into the denser $c(2 \times 2)$ structure [at $\Theta_{0} \approx 0.22 \mathrm{ML}$ (monolayers)], as observed by low energy electron diffraction [9]. The new peak (at $-210 \pm$ $20 \mathrm{meV}$ ) is due to $\mathrm{Rh}$ atoms in the $\mathrm{C}_{2,4}$ configuration (shown in the bottom panel of Fig. 1(a)] and is therefore named $\mathrm{Rh}_{2,4}$.

We now focus on the behavior of the integrated intensities of the $\mathrm{Rh}_{0}, \mathrm{Rh}_{1,4}$, and $\mathrm{Rh}_{2,4}$ peaks as a function of oxygen coverage, shown in Fig. 1(b). We show in the following that these functions contain detailed information on the global kinetics of site occupation during chemisorption.

First of all we note that the signature of the initial adsorption site is directly given by the derivative $\left(\partial \mathrm{Rh}_{0}\right) /$ $(\partial \Theta)$ calculated at low coverage, as we describe hereafter. As shown in Fig. 1(b), upon starting oxygen exposure, $\mathrm{Rh}_{1,4}$ grows linearly with increasing oxygen coverage, at the expense of the $\mathrm{Rh}_{0}$ intensity. This is a consequence of a conservation rule which must hold for surface atoms. For each new oxygen atom adsorbed in the $\mathrm{C}_{1,4}$ configuration, the intensity contribution of four surface $\mathrm{Rh}$ atoms moves from $\mathrm{Rh}_{0}$ to $\mathrm{Rh}_{1,4}$. From this simple observation, it follows directly that for initial adsorption in a fourfold site we must have $\left(\partial \mathrm{Rh}_{0}\right) /(\partial \Theta)=-4$. A fit of the data gives $\left(\partial \mathrm{Rh}_{0}\right) /(\partial \Theta) \approx-4.2$, clearly confirming the adsorption site of $\mathrm{O}$ on $\mathrm{Rh}(100)$. We will show in the following that this link between the initial derivative and the adsorption site symmetry generally holds for atomic chemisorption on transition metals.

We note that the binding energy shift of the $\mathrm{Rh}_{1,4}$ component with respect to $\mathrm{Rh}_{0}$ is $\Delta E_{1,4}=+220 \mathrm{meV}$, while the shift of the $\mathrm{Rh}_{2,4}$ peak is $\Delta E_{2,4}=+435 \mathrm{meV}$. Therefore, within the experimental error

$$
\Delta E_{2,4}=2 \Delta E_{1,4}
$$

We repeated the experiment for the $\mathrm{O} / \mathrm{Rh}(111)$ system, taking a sequence of about 150 SCL spectra during oxygen chemisorption, and found that three new surface peaks arise in sequence upon increasing oxygen exposure. Oxygen is known to adsorb on $\mathrm{Rh}(111)$ in threefold sites $[8,10]$ (i.e., in a $\mathrm{C}_{1,3}$ configuration using our notation). In this case, for each new adsorbed oxygen atom, the intensity contribution of three $\mathrm{Rh}$ atoms should shift from $\mathrm{Rh}_{0}$ to $\mathrm{Rh}_{1,3}$. Experimentally we find $\left(\partial \mathrm{Rh}_{0}\right) /(\partial \Theta)=-3$, a value that again pinpoints with a high level of confidence the site of adsorption. Generalizing our results, for initial adsorption on a site with $j$-fold symmetry, we should have

$$
\frac{\partial \mathrm{Rh}_{0}}{\partial \Theta}=-j \quad(j=1, \ldots, n),
$$

where $n$ represents the maximum $n$-fold site symmetry on the surface of choice. In principle we may expect also $\left(\partial \mathrm{Rh}_{1, j}\right) /(\partial \Theta)=j$. Usually however, photoelectron diffraction effects may influence $\mathrm{Rh}_{1, j}(\Theta)$ and obscure its initial derivative property, while $\mathrm{Rh}_{0}$ is only marginally affected because emission from bare surface atoms is free from focusing effects generated by adparticles [11]. A survey of the recent SCLS (surface core level shift) literature $[12,13]$ shows that Eq. (2) holds in all the cases where approximate values of the initial derivative can be inferred from the published data. The simple rule expressed by Eq. (2) represents therefore a completely new and straightforward experimental method for determining the symmetry of the initial site of chemisorption.

With reference to $\mathrm{Rh}_{0}$, the binding energy shift of the first peak $\mathrm{Rh}_{1,3}$ appearing upon oxygen exposure is $\Delta E_{1,3}=+345 \mathrm{meV}$. The shifts of the second and third peaks are $\Delta E_{2,3}=+780$ and $\Delta E_{3,3}=+1120 \mathrm{meV}$, respectively.

Again, within the experimental error bars we have

$$
\Delta E_{i, 3} \approx i \Delta E_{1,3} \quad(i=1,2,3) .
$$

In other words, for both $\mathrm{O} / \mathrm{Rh}(100)$ and $\mathrm{O} / \mathrm{Rh}(111)$, the energy shifts of the SCL peaks occurring with increasing coverage are multiples of the energy shift of the first peak. We can generalize Eqs. (1) and (3) into the following:

$$
\Delta E_{i, j} \approx i \Delta E_{1, j} \quad(i=1, \ldots, j ; j=3,4),
$$

where $\Delta E_{i, j}$ is the SCL energy shift of a substrate metal atom in the adsorption configuration $\mathrm{C}_{i, j}$, measured with respect to the clean surface core level binding energy.

A survey of the literature [14-17] shows that Eq. (4) holds with good approximation for all the atomic adsorption systems tested, although its generality had remained unnoticed so far. Table I summarizes our present data as well as previous work. The result expressed by Eq. (4) demonstrates an additivity property of the energy shift of a transition metal SCL caused by the individual contributions of different fractions of adsorbate atoms.

This property is clearly an initial state effect [5] originating from the electronic structure of the system. Final state effects, due to screening processes of the core hole, are known to be important for a quantitative understanding of the experimental values of SCLSs [18]. Nevertheless, although they are by no means negligible, extensive DFT calculations [15] have shown that for transition metal adsorption systems they are not large enough to obscure initial state trends, as first pointed out by Citrin et al. [19].

We conclude that time resolved sequences of SCL spectra such as the ones reported in Fig. 1(b) and interpreted through Eqs. (2) and (4) represent dynamical fingerprints of instantaneous configuration populations of adsorbates forming on a surface during chemisorption [20]. 
TABLE I. Core level shifts observed in different $\mathrm{C}_{i, j}$ configurations. The last column reports the linear correlation coefficients $R$.

\begin{tabular}{lcccc}
\hline \hline Threefold & $i=1 ; j=3$ & $i=2 ; j=3$ & $i=3 ; j=3$ & $R$ \\
\hline $\mathrm{O} / \mathrm{Rh}(111)^{\mathrm{a}}$ & 345 & 780 & 1120 & 0.998 \\
$\mathrm{O} / \mathrm{Ru}(001)^{\mathrm{b}}$ & 386 & 776 & 1326 & 0.995 \\
$\mathrm{O} / \mathrm{Ru}(10 \overline{1} 0)^{\mathrm{c}}$ & 395 & 695 & $\cdots$ & 0.997 \\
$\mathrm{O} / \mathrm{W}(111)^{\mathrm{d}}$ & $317^{\mathrm{e}}$ & 660 & 1050 & 0.999 \\
Fourfold $^{\mathrm{O} / \mathrm{Rh}(100)^{\mathrm{a}}}$ & $i=1 ; j=4$ & $i=2 ; j=4$ & & $R$ \\
\hline \hline
\end{tabular}

${ }^{\mathrm{a}}$ This work and Ref. [15].

${ }^{\mathrm{b}}$ Reference [16].

${ }^{\mathrm{c}}$ Reference [14].

${ }^{\mathrm{d}}$ Reference [17].

e (e) On W(110) and at low coverage oxygen resides in one of the corners of the rhombus is a pseudo-threefold site, forming two inequivalent bonds with $\mathrm{W}$ atoms. The value reported for the $\mathrm{C}_{1,3}$ configuration is a weighted average of two different core level shifts.

Figure 2(a) shows changes of SCLS's induced by oxygen in the various configurations $\mathrm{C}_{i, j}$ formed on $\mathrm{Rh}(100)$ and $\mathrm{Rh}(111)$. These changes are measured, in each case, with respect to the energy of the surface peak of the corresponding clean surface. Besides the remarkable linearity of the shifts, we note that the slopes formed for the two crystallographic orientations are similar, yet clearly different (870 and $1120 \mathrm{meV} / \mathrm{ML}$, respectively). This result must have deep roots at the fundamental level, as its origin is a direct consequence of the local changes of electronic structure induced by the chemisorption process. To unravel these implications, we have carried out DFT calculations [22] for the $\mathrm{O} / \mathrm{Rh}(111)$ and $\mathrm{O} / \mathrm{Rh}(100)$ electronic structures at $0.25,0.50$, and $1.00 \mathrm{ML}$ oxygen coverages.

In Fig. 2(b) we show a plot of the changes of SCLSs $\left(\Delta\right.$ SCLS) of $\mathrm{Rh}$ atoms in different $\mathrm{C}_{i, j}$ configurations (each measured from the respective clean surface peak),
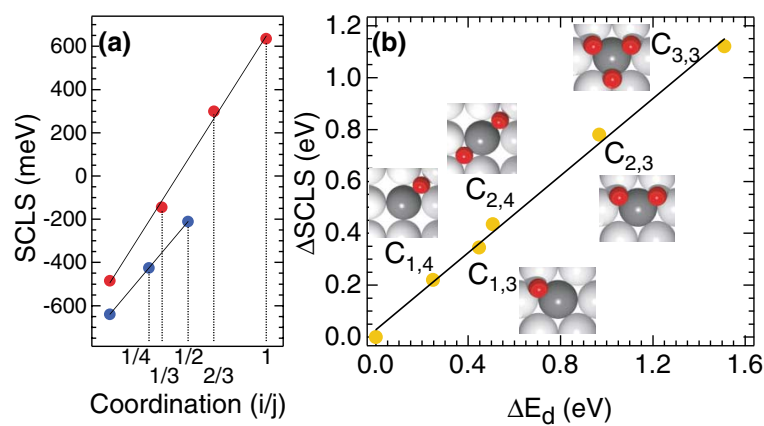

FIG. 2 (color). (a) Surface core level shifts induced by oxygen adsorption on $\mathrm{Rh}(111)$ and $\mathrm{Rh}(100)$ versus the $\mathrm{Rh}-\mathrm{O}$ coordination $i / j$. (b) Core level shifts for rhodium atoms in different $C_{i, j}$ configurations, versus the calculated atom-projected shift of the $d$-band center. The shifts are measured from the corresponding clean surface peak. versus the calculated atom-projected shift of the $d$-band center, which summarizes the results for both systems. The graph displays a striking linear behavior and proves that the correlation between the SCLSs and the $d$-band center is very strong and can be regarded as a general phenomenon.

Previous theoretical work $[24,25]$ has clearly demonstrated that any induced change of the $d$-band center measures a change in chemical reactivity of $d$-metal surface atoms. Unfortunately, the task of measuring the surface $d$-band density of states and disentangling it from the bulk contribution is practically impossible. However, our present results show that for a transition metal, the SCLS is directly proportional to the surface $d$-band center shift, as shown in Fig. 2(b). We have found therefore for the first time, a quite general and easily accessible spectroscopic determination of local changes of surface chemical reactivity.

As a further proof of this concept we carried out DFT calculations to estimate the adsorption energy of nitric oxide on the clean and oxygen precovered $\mathrm{Rh}(100)$ and $\mathrm{Rh}(111)$ surfaces. This quantity is important to understand the NO reduction process in the automotive threeway catalyst [26]. The results are plotted in Fig. 3 versus the measured oxygen induced SCLSs. The linear correlation coefficient is strikingly close to 1 strongly supporting the idea that SCLSs provide a measure of changes of local surface reactivity.

Since the sequential occupation of adsorption sites exhibits a rich phenomenology, the question arises on the behavior of SCL energy shifts for molecular adsorption and for other adsorption geometries. To address this issue we have carried out the experiment on the $\mathrm{CO} / \mathrm{Pt}(111)$ adsorption system. Upon adsorption of $\mathrm{CO}$ on $\operatorname{Pt}(111)$, the initial $4 f_{7 / 2}$ clean surface core level peak starts decreasing while a new peak grows at a shift of $\Delta E_{1,1}=+1410 \pm 25 \mathrm{meV}$. The initial derivative $\left(\partial \mathrm{Pt}_{0}\right) /$ $(\partial \Theta)$ is -1 in excellent agreement with the well-known on-top $\left(\mathrm{C}_{1,1}\right.$ coordination $)$ adsorption site [13]. At higher

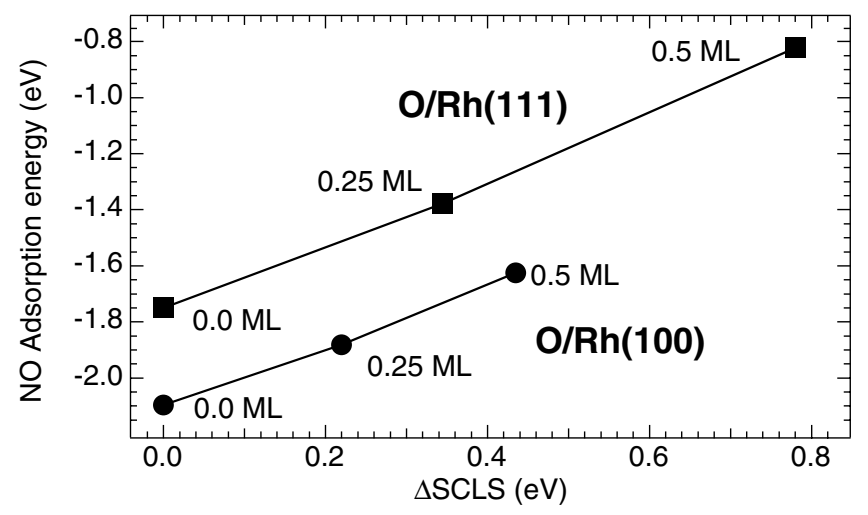

FIG. 3. Calculated NO adsorption energies versus experimental changes of surface core level binding energy for $\mathrm{Rh}(111)$ and $\mathrm{Rh}(100)$ surfaces with different amounts of preadsorbed oxygen. 
coverages also bridge sites $\left(\mathrm{C}_{1,2}\right.$ coordination $)$ start populating, originating a peak in our spectra at $\Delta E_{1,2}=$ $+770 \pm 25 \mathrm{meV}$ (i.e., $\Delta E_{1,2} \approx 1 / 2 \Delta E_{1,1}$ ).

Recent experiments and DFT calculations [27] for CO adsorption on $\mathrm{Rh}(111)$ find a SCL of the $\mathrm{Rh} 3 d_{5 / 2}$ peak at $\Delta$ SCLS $\approx 790 \mathrm{meV}$ for on-top adsorption and at $\approx 280 \mathrm{meV}$ (i.e., $\approx 1 / 3 \Delta \mathrm{SCLS}$ ) for subsequent threefold adsorption. It appears therefore that for molecular adsorption on transition metals the following approximate relationship holds:

$$
\Delta E_{1, j} \approx \frac{1}{j} \Delta E_{1,1}
$$

A survey of the literature $[27,28]$ shows that this finding is also quite general although it had remained unnoted.

Equation (5), together with Eqs. (2) and (4), completes the new set of rules we have found to hold for SCL, which completely describes the time and/or coverage evolution of atomic and molecular adsorption configurations in a chemisorption experiment.

Summarizing we have shown that high resolution SCL sequences of spectra of $d$-band metals contain a wealth of quantitative information which allow the observation of the dynamical evolution of a chemisorption process. Through DFT calculations we have also established a clear link between SCLS and adsorbate-induced local changes of surface chemical reactivity. Our findings represent a very valuable method for interpreting the evolution of the elementary kinetic phenomena taking place in a surface chemical reaction. The direct in situ and realtime measurement of local reactivity changes may prove decisive for an in-depth understanding of many surface elementary processes.

We are grateful to F. Rosei for helpful discussions and for continuous support. We acknowledge financial support from the EU through Contract No. HPRN-CT-2002-00170 and from the MIUR under the Program PRIN2003.

*Corresponding author.

Email address: rosei@elettra.trieste.it

[1] G. Ertl, Adv. Catal. 45, 1 (2000).

[2] H.C. Kang and W. H. Weinberg, Chem. Rev. 95, 667 (1995).

[3] F. Rosei and R. Rosei, Surf. Sci. 500, 395 (2002).

[4] B. Hammer and J. K. Nørskov, Adv. Catal. 45, 71 (2000).

[5] N. Mårtensson and A. Nilsson, Applications of Synchrotron Radiation, edited by W. Eberhardt, Springer Series in Surface Sciences Vol. 35 (Springer, Berlin, 1995), p. 65.

[6] A. Baraldi, G. Comelli, S. Lizzit, M. Kiskinova, and G. Paolucci, Surf. Sci. Rep. 49, 169 (2003).

[7] All the spectra have been fitted by using Doniach-Sunjic line shapes described in Ref. [6].

[8] H. Over, Prog. Surf. Sci. 58, 249 (1998).
[9] A. Baraldi, V. R. Dhanak, G. Comelli, K. C. Prince, and R. Rosei, Phys. Rev. B 56, 10511 (1997).

[10] M.V. Ganduglia-Pirovano and M. Scheffler, Phys. Rev. B 59, 15533 (1999).

[11] D. P. Woodruff and A. M. Bradshaw, Rep. Prog. Phys. 57, 1029 (1994).

[12] A. Beutler et al., Surf. Sci. 371, 381 (1997).

[13] O. Björneholm et al., Surf. Sci. 315, L983 (1994).

[14] A. Baraldi, S. Lizzit, and G. Paolucci, Surf. Sci. 457, L354 (2000).

[15] M. V. Ganduglia-Pirovano et al., Phys. Rev. B 63, 205415 (2001).

[16] S. Lizzit et al., Phys. Rev. B 63, 205419 (2001).

[17] D. M. Riffe and G. K. Wertheim, Surf. Sci. 399, 248 (1998).

[18] M.V. Ganduglia-Pirovano, J. Kudrnovsky, and M. Scheffler, Phys. Rev. Lett. 78, 1807 (1997).

[19] P. H. Citrin, G. K. Wertheim, and Y. Bear, Phys. Rev. Lett. 41, 1425 (1978).

[20] Other spectroscopic methods (energy loss and infrared [21]) also yield information on time dependent site occupation. By measuring directly a physical property (e.g., vibrational frequencies) they also determine the adsorbate's registry. As opposed to these traditional methods, our technique additionally measures the distribution of atoms which share multiple bonds with other adparticles.

[21] D. P. Woodruff and T. A. Delchar, Modern Techniques of Surface Science (Cambridge University Press, Cambridge, 1994), 2nd ed.

[22] The DFT calculations were performed using the DACAPO code (http://www.fysik.dtu.dk/CAMPOS), where the Kohn Sham equations are solved in a plane wave basis restricted by a kinetic energy cutoff of $25 \mathrm{Ry}$. We represented the core electrons of all the atoms with Vanderbilt nonlocal ultrasoft pseudopotentials [23] and used the revised Perdew, Burke, and Ernzerhof. This is a commonly used generalized gradient approximation for the exchange and correlation energy of electrons. Samplings of $18 k$ points for a $\mathrm{Rh}(111)$ surface and $16 k$ points for a $\mathrm{Rh}(100)$ surface were used for Brillouin zone integration together with a Fermi smearing of $\sigma=0.1 \mathrm{eV}$. All total energies were extrapolated to zero temperature. The two $\mathrm{Rh}$ surfaces were modeled with a slab of three atomic layers with $2 \times 2,2 \times 1$, and $1 \times 1[\mathrm{Rh}(111)]$ and $2 \times 2$ [Rh(100)] surface cells. Adsorption was allowed only at one side of the slab and the two topmost metal layers were relaxed.

[23] D. Vanderbilt, Phys. Rev. B 41, 7892 (1990).

[24] B. Hammer and J. K. Nørskov, Nature (London) 376, 238 (1995).

[25] M. Mavrikakis, B. Hammer, and J. K. Nørskov, Phys. Rev. Lett. 81, 2819 (1998).

[26] M. J. P. Hopstaken and J.W. Niemantsverdriet, J. Chem. Phys. 104, 3058 (2000).

[27] M. Birgersson, C. O. Almbladh, M. Borg, and J. N. Andersen, Phys. Rev. B 67, 045402 (2003).

[28] F. Strisland, A. Ramstad, T. Ramsvik, and A. Borg, Surf. Sci. 415, L1020 (1998). 